K. Lee, G.C. Temes, F. Maloberti: "Noise-Coupled Multi-Cell Delta-Sigma ADCs"; IEEE International Symposium on Circuits and Systems, ISCAS 2007, New Orleans, 27-30 May 2007, pp. 249-252.

(C)20xx IEEE. Personal use of this material is permitted. However, permission to reprint/republish this material for advertising or promotional purposes or for creating new collective works for resale or redistribution to servers or lists, or to reuse any copyrighted component of this work in other works must be obtained from the IEEE. 


\section{Noise-Coupled Multi-Cell Delta-Sigma ADCs}

\author{
Kyehyung Lee and Gabor C. Temes \\ School of Electrical Engineering and Computer Science \\ Oregon State University \\ Corvallis, Oregon, USA \\ \{leekye, temes\}@eecs.oregonstate.edu
}

Abstract-Noise-coupled multi-cell delta-sigma ADCs are presented. By providing quantization noise coupling between identical delta-sigma ADCs, one can effectively raise the order of the modulator. Cross-coupled noise injection also provides an efficient way to realize higher-order time-interleaved ADCs.

\section{INTRODUCTION}

In an earlier paper [1], a multi-cell delta-sigma ADC was introduced to increase the robustness and programmability of delta-sigma ADC. The key of the method is the capability to raise the order of a noise transfer function by the crosscoupled injection of the quantization noise [2]. In this work, we propose two types of noise coupling in multi-cell deltasigma ADCs, and extend the operation to time-interleaved (TI) ADCs. The noise coupling between different modulator cells (cross-enhanced delta-sigma ADC) or within each modulator cell (self-enhanced delta-sigma ADC) raises the effective order of the modulator by one or more, depending on the applied coupling filter, without aggravating the stability conditions. This is due to the statistical independence of quantization noise samples, which can be assumed under busy signal conditions and for multi-bit quantization. Then the injected noise acts as merely as dither, removing harmonic spurs. Thus, the stability condition of the original modulator is preserved.

\section{NOISE COUPLING IN MULTI-CELL ADCS}

\section{A. First-order noise coupling}

The order of a delta-sigma modulator (DSM) can be enhanced by self-coupled noise injection, shown in the scheme of Fig. 1. A delayed replica of the quantization error is fed back into the modulator to be shaped by the noise

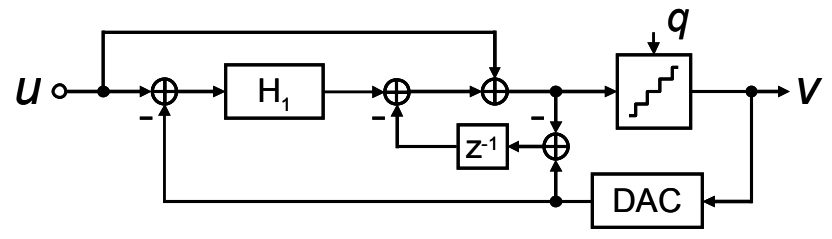

Fig. 1. DSM with self-coupled noise injection.

\author{
Franco Maloberti \\ Department of Electronics \\ University of Pavia \\ 27100 Pavia, Italy \\ franco.maloberti@unipv.it
}

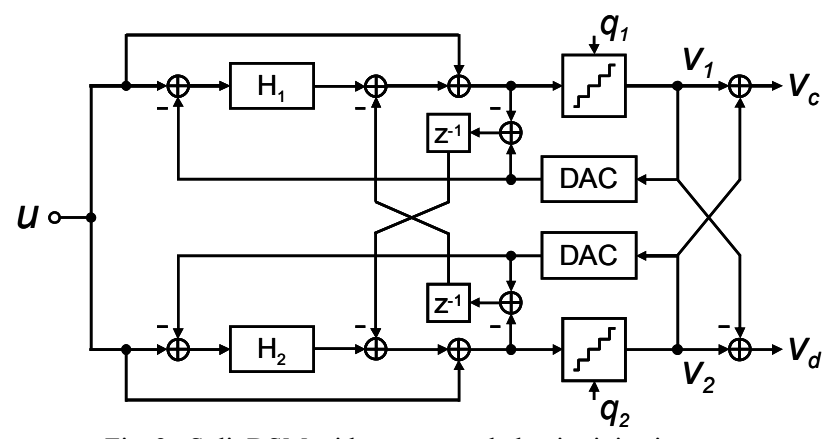

Fig. 2. Split DSM with cross-coupled noise injection.

transfer function (NTF). Since this increments the NTF by an extra $\left(1-z^{-1}\right)$ factor, the order of the modulator is increased by one. The structure can be duplicated, to provide the sum of the two outputs for signal processing, and their difference for calibration purposes [3].

For duplicated modulators, the noise injection can be cross-coupled as shown in Fig. 2. Since the coupled quantization noises are injected at the quantizer inputs, any error signals in the coupling branches will be suppressed by the gain of the loop filter. Therefore, mismatches between DSM cells minimally affect cross-coupled delta-sigma ADCs. Self-enhanced delta-sigma ADCs do not suffer from any mismatches between DSM cells.

\section{B. Second and higher-order noise coupling}

The noise-coupling concept can be extended to higherorder coupling branches. By designing the coupling filter properly, the order of modulator can be increased by two or more. Second-order coupling can be implemented using a transfer function $\left(2 z^{-1}-z^{-2}\right)$ in the coupling branch, as shown in Fig. 3. The practical implementation of the cross coupling typically uses added capacitors that worsen the feedback factor of the opamps used. The additional power consumption required is a small price to pay for a significant benefit. Generally, the order of modulator can be increased by $n$ using a coupling block from DSM cell $j$ to DSM cell $i$ (Fig. 4) with a transfer function

This research was funded by the NSF Center for the Design of Analog/Digital Integrated Circuits (CDADIC). 


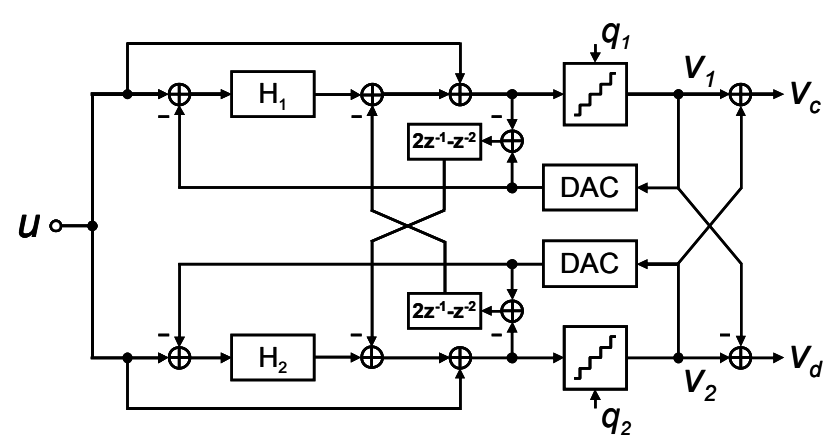

Fig. 3. Split DSM with second-order cross-coupled noise injection.

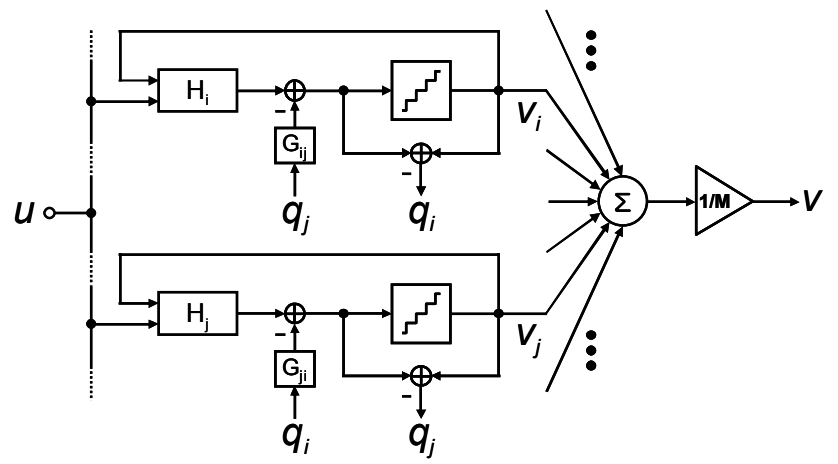

Fig. 4. Generalized split DSM with cross-coupled noise injection.

$$
G_{i j}=z^{-1} \sum_{k=0}^{n-1}\left(1-z^{-1}\right)^{k} .
$$

Ideally, cross-enhanced and self-enhanced couplings provide equivalent performance improvements. But selfenhanced noise coupling is not subject to injected quantization noise leakage due to NTF mismatch, and is hence more suitable for programmable multi-cell DSMs.

\section{NOISE COUPLING IN TIME-INTERLEAVED ADCS}

Noise coupling can be used also in time-interleaved (TI) DSMs [4]. Any circuit errors on the coupling branch are suppressed by the loop filter gain, and the overall performance is insensitive to coupling errors, as in multi-cell DSMs. But there is a significant difference between the performances of two types of noise coupling in TI deltasigma ADCs, unlike in multi-cell delta-sigma ADCs.

\section{A. Self-enhanced dual-channel TI delta-sigma ADC}

If the signal transfer function satisfies STF $=1$ as is the case for the low distortion architecture described in [5], and NTF is the noise transfer function of each channel modulator, then the dual-channel TI modulator output is

$$
V(z)=U(z)+\left(1-z^{-2}\right) \operatorname{NTF}\left(z^{2}\right) Q(z) .
$$

Self-coupled quantization noise injection changes the original NTF to $\left(1-z^{-2}\right) \cdot N T F$. Thus, the order of the NTF is increased, and an out-of-band zero at $z=-1$ appears. The additional zero doubles the low-frequency quantization noise, which results in a $6 \mathrm{~dB}$ SNDR degradation.

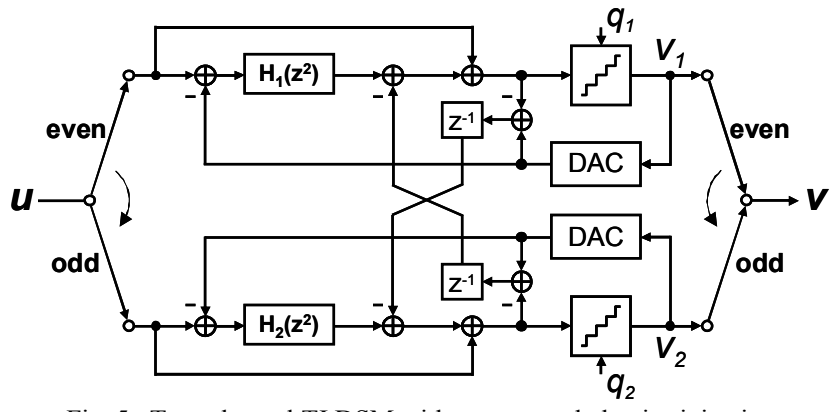

Fig. 5. Two-channel TI DSM with cross-coupled noise injection.

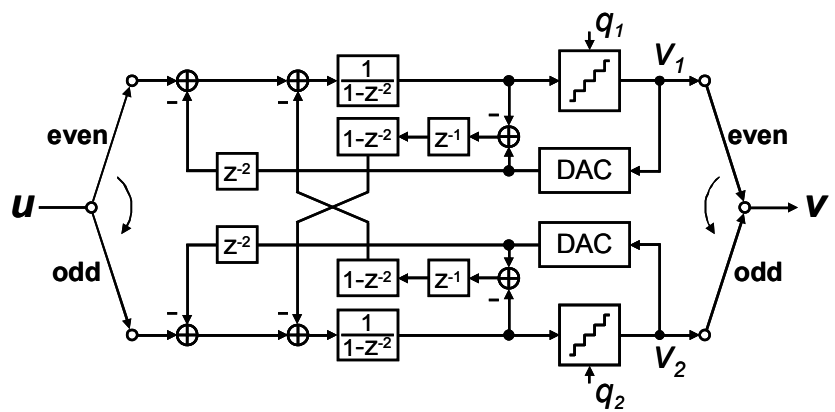

Fig. 6. Two-channel TI DSM with modified cross-coupled noise injection

\section{B. Cross-coupled dual-channel TI delta-sigma ADC}

The output signal of the dual-channel TI modulator with cross-coupled quantization noise injection is given by

$$
V(z)=U(z)+\left(1-z^{-1}\right) N T F\left(z^{2}\right) Q(z) .
$$

Unlike self-coupled noise injection, cross-coupled noise injection can enhance the order of NTF without introducing additional out-of-band zeros. Therefore, it provides a $6 \mathrm{~dB}$ SNDR improvement compared to self-coupled noise injection. Observe that the scheme of Fig. 5 with loop filters $H\left(z^{2}\right)=z^{-2} /\left(1-z^{-2}\right)$ gives an $N T F=\left(1+z^{-1}\right) \cdot\left(1-z^{-1}\right)^{2}$. A problem in the implementation of the scheme of Fig. 5 is the combination of many analog signals before the quantizer. However, it is possible to move the point of noise injection to the input of the $H\left(z^{2}\right)$ block, as shown in Fig. 6, where $H\left(z^{2}\right)$ was split into a double delay and a $1 /\left(1-z^{-2}\right)$ block. Moving the injection as shown in Fig. 6 avoids the need for combining analog signals at the quantizer input, except for the input feed-forward path, not shown in Fig. 6. Note also that the coupling can be partially performed in the digital domain.

Simulated NTFs without noise coupling and with selfand cross-coupled noise injection are compared in Fig. 7, where the original NTF was a second-order one, and ideal circuit elements were assumed. Fig. 8 shows the simulated power spectral densities (PSDs) and the integrated PSDs for self-enhanced and cross-enhanced dual-channel TI DSMs. Reasonable assumptions were made for thermal noise and other non-ideal effects. The $6 \mathrm{~dB}$ difference between the curves is evident for frequencies over $1 \%$ of the sampling frequency. 


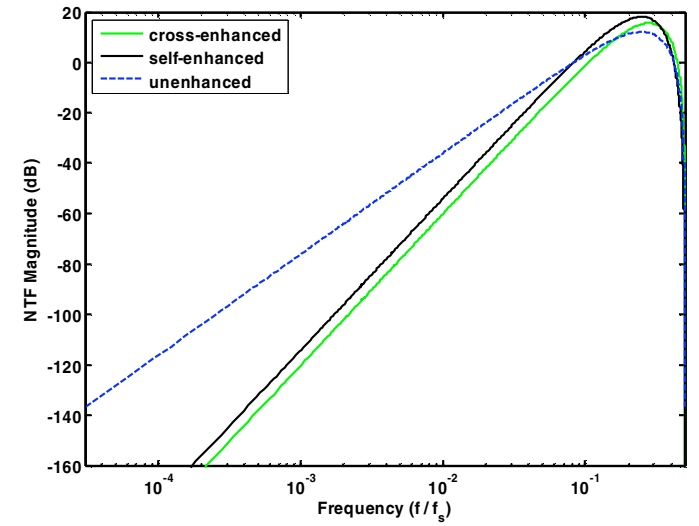

Fig. 7. Comparison of NTFs for dual-channel TI DSMs, without and with first-order noise coupling.

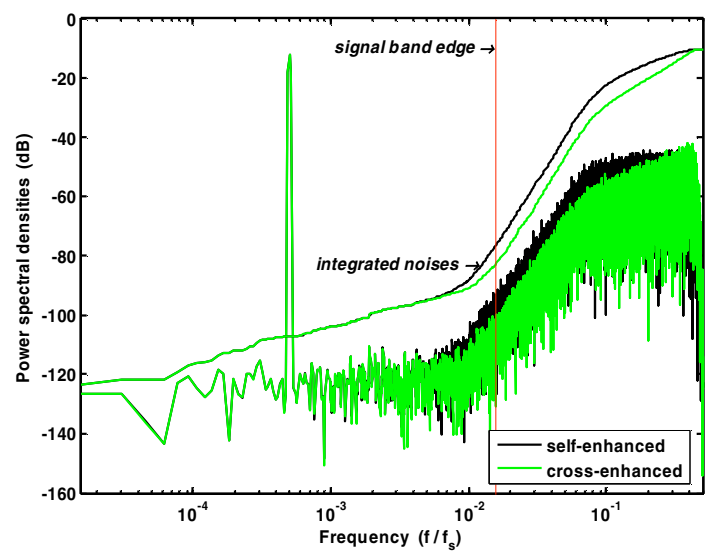

Fig. 8. Simulated PSDs for dual-channel TI DSMs with self-enhanced noise coupling $(\mathrm{SNDR}=66.6 \mathrm{~dB})$ and cross-enhanced noise coupling $(\mathrm{SNDR}=$ $72.8 \mathrm{~dB})$.

\section{Second-order noise coupling in dual-channel TI DSMS}

The performance gap between self-coupled and crosscoupled dual-channel TI delta-sigma ADCs employing second-order coupling branches is $12 \mathrm{~dB}$, due to the additional double zeros of the NTF introduced in secondorder self-enhanced noise coupling. The NTF curves for second-order noise coupling are shown in Fig. 9. In general, it is advantageous to raise the order of the NTF in TI lowpass delta-sigma $\mathrm{ADC}$ without creating additional out-ofband zeros. This can be achieved with higher-order crosscoupled quantization noise injection.

\section{SimUlation RESUlTS}

To verify the theoretical results, a dual-channel TI DSM was extensively simulated using Simulink and Matlab [6]. The low-distortion architecture proposed in [5] was selected for simulation, since it greatly reduces the dynamic range of the integrators, and relaxes the linearity and slew-rate requirements of the opamps. Analog circuit errors, such as finite opamp gain, capacitor mismatches in the feedback DACs, modulator coefficient deviations, quantizer offsets,

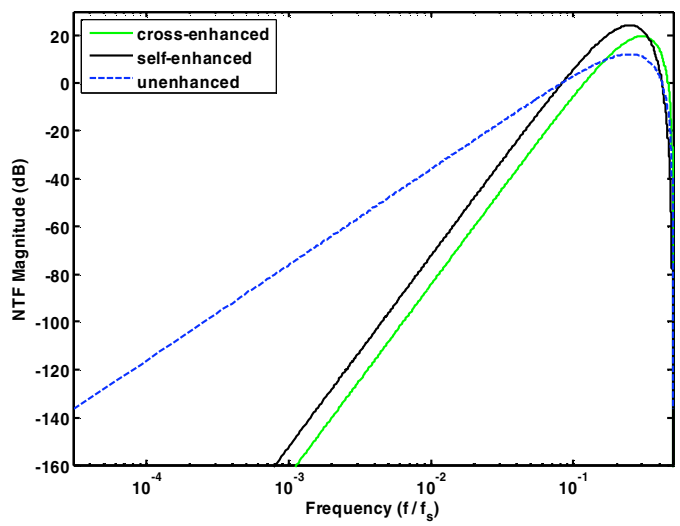

Fig. 9. Comparison of NTFs for a dual-channel TI DSM, without and with second-order noise coupling.

thermal noise, and clock jitter were included in the models used. The unenhanced modulator without noise coupling had a second-order NTF, and it used a nine-level quantizer. The dual-channel operation reduces the operation speed of each channel by half, and the oversampling ratio (OSR) of each channel modulator was 16 , which makes the effective OSR of the overall TI modulator 32. First-order noise coupling was applied, both in a self-coupled and cross-coupled structure.

\section{A. SNDR variation with input amplitude}

Fig. 10 illustrates the SNDR performance variation with input signal amplitude. The peak SNDR was obtained for a -3 dBFS input for the cross-enhanced TI DSM, while it occured for a -2 dBFS input for the self-enhanced TI DSM, and for a -1 dBFS input for the unenhanced TI DSM. The opamp gains used were $50 \mathrm{~dB}$ and $40 \mathrm{~dB}$ for the first and second integrator, respectively.

\section{B. SNDR variation with opamp gain}

Fig. 11 illustrates the SNDR performance variation with finite opamp DC gain values between $17.5 \mathrm{~dB}$ and $67.5 \mathrm{~dB}$. The opamp gain for the first integrator was $10 \mathrm{~dB}$ higher than that for the second integrator in each channel, and the

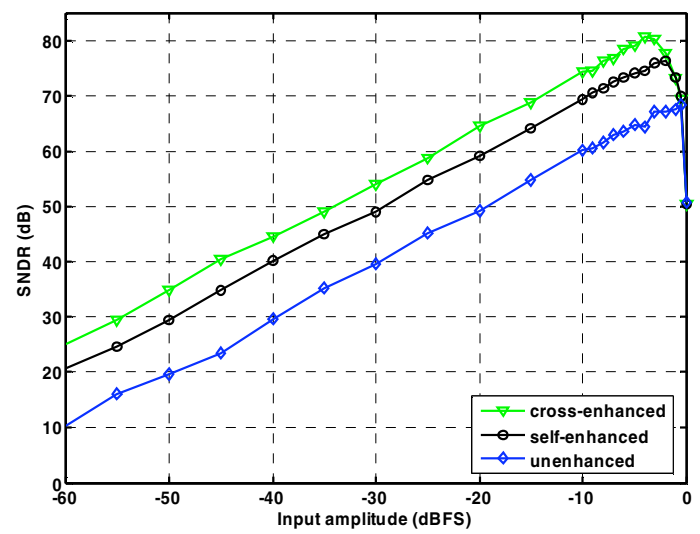

Fig. 10. SNDR performance variation with input signal amplitude for dualchannel TI DSM without noise coupling and with first-order noise coupling. 


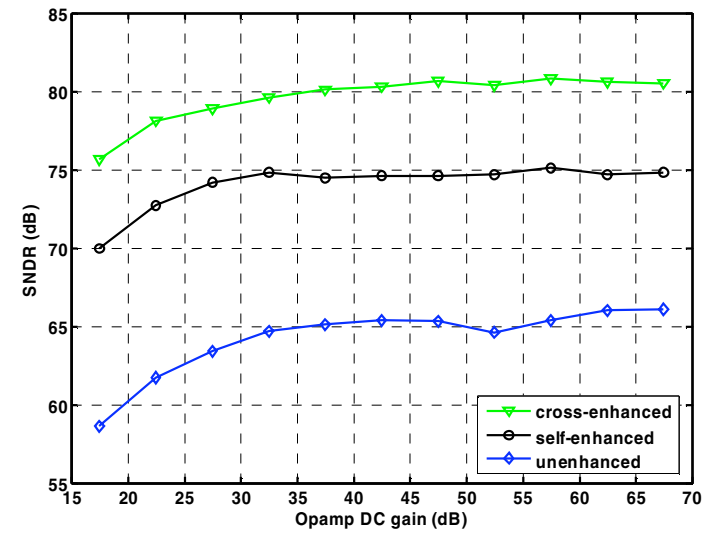

Fig. 11. Performance variation with opamp gain for a dual-channel TI DSM without and with first-order noise coupling. Perfectly matched channels were assumed.

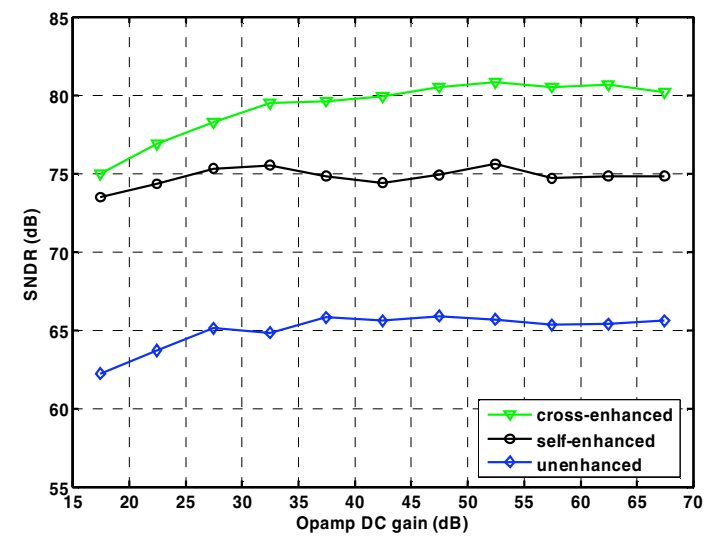

Fig. 12. Performance variation with opamp gain for a dual-channel TI DSM, without and with first-order noise coupling. A 5\% channel mismatch was assumed.

average value of all four opamp gains was swept. In this sweep, the opamp gain mismatch between dual channels was not included. Each modulator shows robust performance under very low opamp DC gains with minor performance degradation. Fig. 12 shows the SNDR variation with opamp gains when a 5\% opamp gain mismatch was assumed between dual channels. As pointed out earlier, noise transfer function deviation due to opamp gain mismatches between channels results in a leakage of injected quantization noise in a cross-coupled TI DSM. But the performance degradation due to this quantization noise leakage does not become significant until the opamp gains become very low $(<35$ $\mathrm{dB})$.

\section{SNDR variation with coefficient errors}

The modulator coefficients on both channels were changed from their nominal values by random errors ranging from $0.05 \%$ to $3.1 \%$, and the variation of the SNDR was simulated. As shown in Fig. 13, no evident performance degradation was observed due to the modulator coefficient drifts in this range for either unenhanced or self-enhanced TI

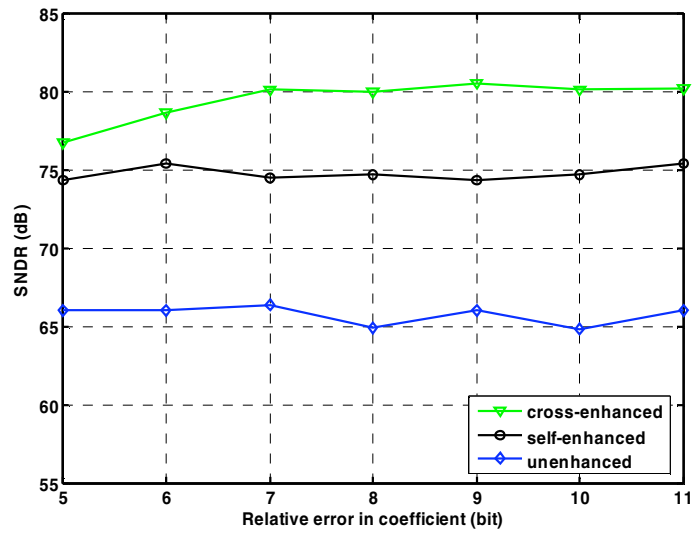

Fig. 13. Performance variation with coefficient error for dual-channel TI DSM without and with first-order noise coupling (5 bit relative error corresponds to a $2^{-5}=3.1 \%$ coefficient error).

delta-sigma ADCs. For cross-enhanced TI DSM, there was no performance deterioration with modulator coefficient deviations up to $0.78 \%$, and $3 \mathrm{~dB}$ SNDR degradation was observed with $3.1 \%$ modulator coefficient errors.

\section{CONCLUSIONS}

New delta-sigma modulator topologies with quantization noise coupling were presented. Two types of noise coupling were described for both multi-cell DSMs and timeinterleaved DSMs. Either self-coupled or cross-coupled noise injection can raise the order of the noise shaping by one or more, without significantly affecting the stability conditions of the original modulator. When self-enhanced noise coupling is applied to a multi-cell DSM, its robustness and flexibility are also preserved. Cross-coupled noise injection provides an efficient way to raise the order of a TI DSM without creating extra out-of-band zeros.

\section{ACKNOWLEDGMENTS}

The authors are grateful to M. Miller and J. Steensgaard for useful discussions.

\section{REFERENCES}

[1] K. Lee and G. C. Temes, "Enhanced split-architecture delta-sigma ADC," Electron. Lett., vol. 42, no. 13, pp. 737-738, Jun. 2006.

[2] K. Lee, M. Bonu, and G. C. Temes, "Noise-coupled delta-sigma ADCs," Electron. Lett., vol. 42, no. 24, pp. 1381-1382, Nov. 2006.

[3] J. McNeill, M. Coln, and B. Larivee, "'Split-ADC' architecture for deterministic digital background calibration of a 16-bit 1-MS/s ADC," IEEE J. Solid-State Circuits, vol. 40, no. 12, pp. 2437-2445, Dec. 2005.

[4] F. Borghetti, C. Della Fiore, P. Malcovati, and F. Maloberti, "Synthesis of the noise transfer function in n-path sigma delta modulators," Proceedings of Advanced A/D and D/A Conversion Techniques and Their Application (ADDA '05), pp. 171-176, 2005.

[5] J. Silva, U. Moon, J. Steensgaard, and G. C. Temes, "Wideband lowdistortion delta-sigma ADC topology," Electron. Lett., vol. 37, no. 12, pp. 737-738, Jun. 2001.

[6] Simulink and Matlab User Manual, The Matworks Inc. 